\title{
Development of a New System for Real-Time Detection of Radon Using Scintillating Optical Fibers
}

\author{
C. S. Monteiro ${ }^{1}$, L. Coelho ${ }^{2}$, S. M. Barbosa ${ }^{1}$ and D. Guimarães ${ }^{1}$ \\ ${ }^{I}$ CSIG - INESC TEC, Campus da FEUP Rua Dr Roberto Frias, 4200-465 Porto, Portugal. \\ ${ }^{2}$ CAP - INESC TEC and Department of Physics and Astronomy, Faculty of Sciences, University of Porto, Rua do Campo Alegre, Porto, \\ Portugal \\ Author e-mail address: diana.f.guimaraes@inesctec.pt
}

\begin{abstract}
A remote sensor for radon continuous measurement using polymeric scintillation optical fibers was developed and evaluated. Successful preliminary results showed detection of natural occurring radon from a container with rocks rich in uranium oxides. OCIS codes: (280.0280) Remote sensing and sensors (280.4788) Optical sensing and sensors
\end{abstract}

\section{Introduction}

Radon (Rn-222) is a natural occurring radioactive gas produced from the decay of Radium-226 which belongs to the radioactive series of Uranium-238 [1]. Radon and its progeny are accepted as the major source of the radiation dose received by the general public with indoor radon accumulation considered as a cause for lung cancer [2]. Moreover, due to its non-reactive characteristics, short half-life (3.8 days) and its widespread occurrence in nature, radon is considered a key environmental tracer and can be used in many geophysical applications: volcanic and tectonic monitoring, atmospheric transport evaluation, accessing submarine groundwater discharges, among others [3].

Despite radon's huge potential as a proxy for geodynamic processes, its practical use is limited by the currently available technology for its measurement. Although a few options are available, most of them lack temporal and spatial resolution, as well as a real-time remote monitoring option that would turn the instruments into devices amenable to be used in robotic platforms.

The use of fiber optics for ionizing radiation detection enabled the development of cost effective, small, portable and spatially flexible sensors [4-6]. Systems based in optical fiber present many advantages over conventional sensors as they are unaffected by electromagnetic interferences, are lightweight and have low transmissions losses through long distances making it suitable for remote access applications [7]. Few works have been published where fiber optics are used for the specific detection of radon either as an optic guide [8], or as the sensor probe constructed from glass fibers through radiation induced attenuation [9], or through scintillation $[10,11]$. The use of polymeric scintillation fibers for this specific application is still scarce but promising [12]. Polymeric scintillation fibers present a large fiber cross-section, facilitating alignment between the fiber and other optic components, increasing the system robustness. A larger core also means a bigger numerical aperture enhancing light transmission and sensibility. This is also an advantage because dust contamination (for example in the fiber coupling end) has now a minor contribution for the signal degradation [4].

In this work, a new optical system prototype for real-time continuous measurement of radon variations is presented. This system consists of encased multi-clad scintillating optical fibers directly coupled to an analog photomultiplier with no multichannel analyzer system needed. A microprocessor Raspberry Pi was implemented to monitor temperature and humidity, to store data, and as a wireless interface for remote access. The design characteristics were optimized using UV radiation and the operating performance of the sensor was evaluated. Finally, the sensor was connected to a radon exhalation setup consisting of a container with rocks rich in natural uranium for measurement of radon gas.

\section{Prototype construction}

The prototype head consists of 4-mm diameter scintillating optical fibers BCF-60 (Saint Gobain Crystals) directly coupled to a photomultiplier (PMT, Hamamatsu H7468-01), as can be seen in Figure 1. The fiber core is doped with a scintillating material that emits photons in the visible range when excited by high energy radiation/particles, generating 7,100 green photons ( $530 \mathrm{~nm}$ ) per each $\mathrm{MeV}$ of interacting alpha, beta or gamma radiation. BCF-60 is sensitive for energies below $5 \mathrm{MeV}$ which makes it suitable to radon and radon progeny detection.

The PMT, that collects the scintillation light operating in the analog mode, was connected to a Raspberry Pi $3 \mathrm{~B}$ with a temperature and humidity sensor and placed in a gas- and light-tight aluminum box $(16 \times 26 \times 12 \mathrm{~cm})$ with mate black walls (Figure 1). The Raspberry Pi runs the PMT algorithm, recording the current measured by the sensor head, as well as the temperature and humidity inside the aluminum box. 


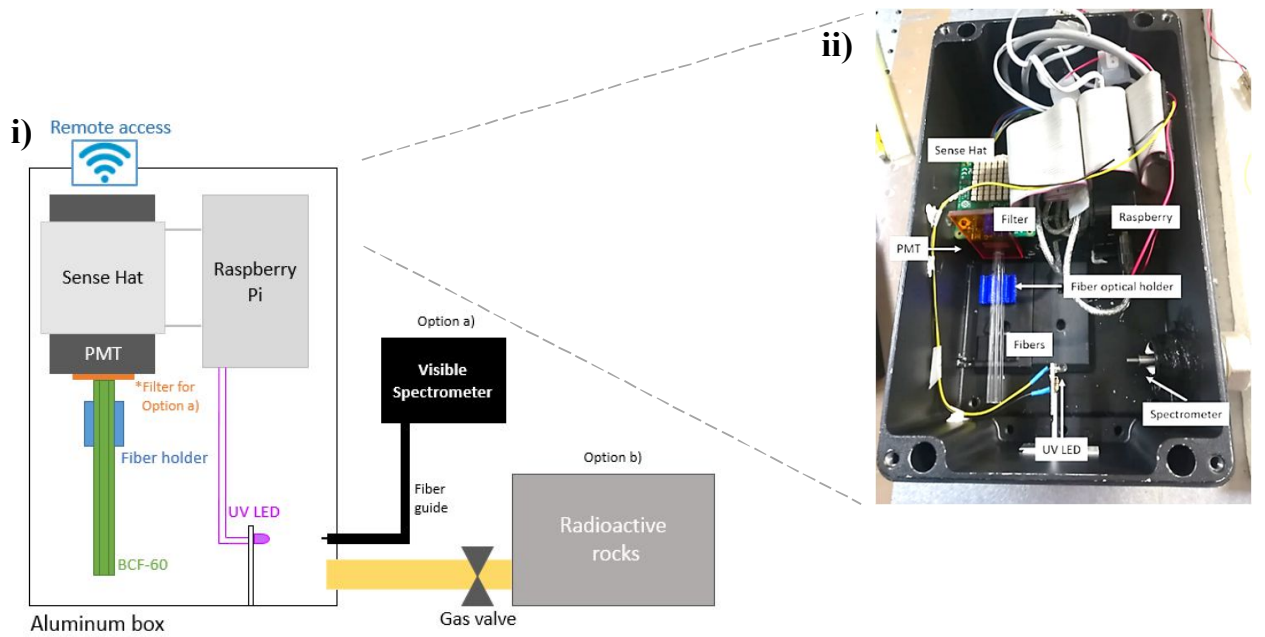

Fig.1. Prototype set-up i) Schematic of set-up with the two modes used: Option a) for optimization and Option b) for radon measurements; ii) Detail inside of aluminum box when operating according to Option a).

The optimization tests were carried out using an UV LED (light emission in the scintillating fiber occurs at the same wavelength as if stimulated by ionizing radiation) with a center wavelength at $470 \mathrm{~nm}$, powered by the Raspberry Pi. A fiber guide connected to a spectrometer (Ocean Optics USB-4000) was placed inside of the aluminum box to monitor the LED intensity. This input signal was used for normalization of the measured PMT signal, making the optimization more robust and independent of source intensity variations. The PMT cathode radiation sensitivity covers the UV range, therefore a filter (orange acrylic, $3 \mathrm{~mm}$ thick) was placed between the PMT window and the fiber-top to maximize the UV blockage while allowing the transmission of the green scintillating photons. All these components can be seen in Figure 1 i) and ii).

The fibers were placed in a 3D printed polymeric holder, designed specifically for the 4-mm diameter fibers in a scheme that maximized the usage of the PMT window, aligned with the center line of the fiber bundle parallel to the axis of the cylindrical PM-tube, as seen in Figure 1.

For radon detection the initial set-up was adapted, as shown in Figure 1 i). The aluminum box was connected to an enhanced confined mode set-up consisting of a container filled with rocks rich in uranium oxides. A metal hose carried the radon gas from the generator container to the aluminum box, distributing the gas to the prototype detector. The gas inlet in the detector box is controlled by a sealed gas valve.

\section{Prototype optimization}

Using one 4-mm diameter BCF-60 fiber, the sensor's response was evaluated for different lengths: 2.5, 5, 7.5 and $10 \mathrm{~cm}$. For two different fiber lengths $(5$ and $10 \mathrm{~cm})$, the sensor performance was assessed for different numbers of fibers: 1, 2 and 3. The volume of fibers was limited by the PMT window size $(\varnothing 8 \mathrm{~mm})$ and the aluminum box dimensions.

The influence of fiber length in the measured signal was studied as presented in Figure 2. The signal increases as the fiber length increases, achieving a 54\% improvement with the $10 \mathrm{~cm}$ fiber when compared to the $2.5 \mathrm{~cm}$ fiber. The sensor response with the variation of the number of fibers is shown in Figure 3, attaining an increase of

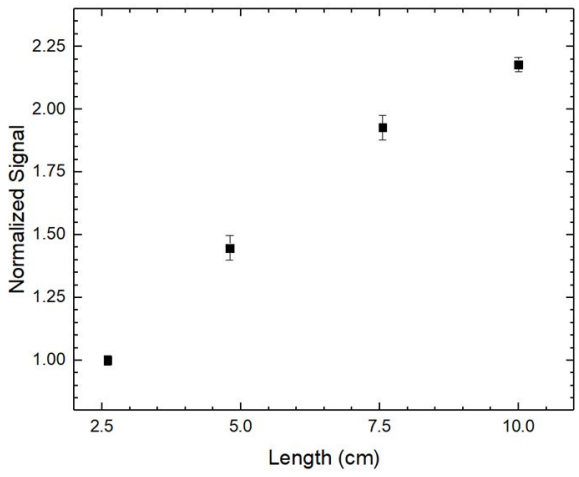

Fig.2. Normalized prototype output signal for different fiber lengths $(n=3)$.

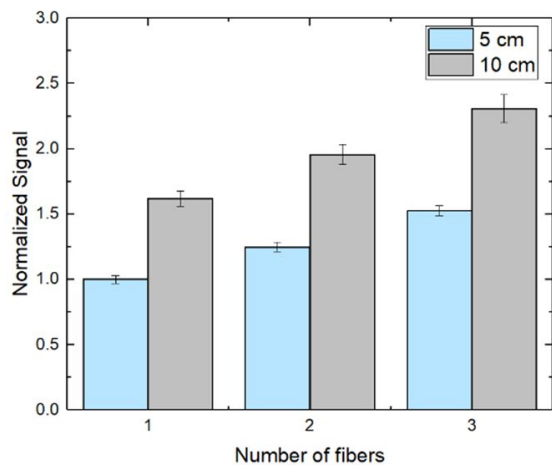

Fig.3. Normalized prototype output signal for different number of fibers, with 5 and $10 \mathrm{~cm}$ length $(n=3)$. 
around $30 \%$ using 3 fibers when compared to 1 fiber, for both lengths studied.

In order to study the repeatability of the system 10 consecutive measurements were taken using 1,2 and 3 fibers of $10 \mathrm{~cm}$ in which the fibers were completely withdrawn and re-inserted inside the system. Results are shown in Table 1. Although the increase in the number of fibers converts into a small decrease in precision, with more measurements falling outside the $5 \%$ interval from the average ( 3 events for 3 fibers $v s 0$ events for 1 fiber) it is also responsible for a significant increase in sensitivity.

Table 1. Repeatability $(n=10)$ for 1,2 and 3 fibers of $10 \mathrm{~cm}$

\begin{tabular}{|c|c|c|c|}
\hline $\begin{array}{c}\text { Number } \\
\text { of Fibers }\end{array}$ & $\begin{array}{c}\text { Average } \\
\text { Normalized } \\
\text { Prototype Signal }\end{array}$ & $\begin{array}{c}\text { Standard } \\
\text { deviation }\end{array}$ & $\begin{array}{c}\text { Relative } \\
\text { Standard } \\
\text { Deviation (\%) }\end{array}$ \\
\hline 1 & 1.05 & 0.03 & 2.9 \\
\hline 2 & 1.25 & 0.04 & 3.2 \\
\hline 3 & 1.40 & 0.05 & 3.6 \\
\hline
\end{tabular}

The sensitivity of the system can be improved by increasing the reflectivity of the fiber end surface (further away from the PMT window), reflecting the incident photons into the fiber core and improving its guidance to the PMT window. For that, four different surface treatments were studied: polished with $16 \mu \mathrm{m}$ grain, optical grade polished (OGP) $\lambda / 10$, OGP combined with a thin layer of PTFE and OGP with a mirror at the surface.

The polishing process follows several steps. The fibers were roughly cleaved and fitted into a custom-made stainless-steel holder that was attached to a jig and sequentially went through a set of 3 polishing plates. The first plate is an iron plate rotating while dripping polishing fluid Logitech Silicon Carbide Powder (600 grit); the second plate is a grooved brass plate rotating while using Logitech Calcinated Aluminum Oxide (1200 grit) polishing fluid; the last plate is an aluminum covered with polyurethane polishing pad plate using Logitech SF1 Polishing Suspension fluid, giving the substrate an OGP.

All the fibers tested had the same length $(10 \mathrm{~cm})$ and were previously optical grade polished at the fiber top that connects to the PMT window. Results are presented in Table 2.

The fiber with the OGP optical grade polished surface presented the lowest signal, when compared to the other surfaces. Using the reflective surface at the fiber-end resulted in increases of $35 \%$ and $45 \%$ for PTFE layer and for the mirror, respectively.

Table 2. Measured signal for different surface treatments $(n=3)$

\begin{tabular}{|c|c|c|c|}
\hline Surface treatment & $\begin{array}{c}\text { Normalized Sensor } \\
\text { Signal (a.u) }\end{array}$ & $\begin{array}{c}\text { Relative Standard } \\
\text { Deviation (\%) }\end{array}$ & Increase (\%) \\
\hline Optical grade polish (OGP) & 1 & 2.4 & - \\
\hline Polished 16 $\mu$ m grain & 1.2 & 3.9 & 19 \\
\hline OGP coated with PTFE & 1.5 & 6.4 & 35 \\
\hline OGP with mirror at fiber end & 1.8 & 3.1 & 45 \\
\hline
\end{tabular}

\section{Radon Measurements}

Preliminary radon measurements were taken according to the schematic presented on Figure 1, option b) and with no mirror at fiber end. The first measurements with this system did not distinguish background from radon signals. However, with the proper threshold in integration and dead time, signal and noise can be distinguished efficiently. Using a dead time and integration time both of $10 \mathrm{~ms}$ it was possible to successfully detect radon build up inside the aluminum box. To process the raw data obtained every 1 second, $1 \mathrm{~h}$ averages were made. A band block FFT filter with the same frequency as the temperature signal was used in the output signal to remove its influence.

As can be seen in Figure 4, the valve started closed and the sensor was measuring the background for a period of 2 days. After, the valve was opened, and the radon built up inside the aluminum box for about 4 days. This build up can be seen in Figure 4 with the increase of the signal. Finally, the valve was closed and a decrease in the signal was attained, that translates in the decrease in radon concentration over the next 7 days. 


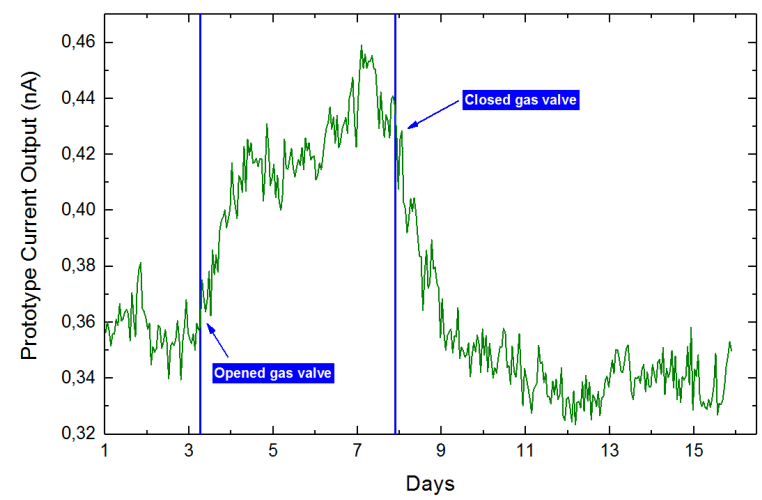

Fig.4. Multi-day radon signal from rocks rich in uranium obtained with this prototype

\section{Conclusions}

A new radon measuring system made of scintillation polymeric fiber optics has been developed and optimized. Optimization showed that, considering the limitations of our system, the maximum sensitivity was achieved using 3 fibers of 4-mm diameter and $10 \mathrm{~cm}$ length and having a mirror in the fiber-end. Precision of this system is mostly within 5\%. This prototype successfully detected radon and using the adequate dead and integration times, was possible to filter noise and efficiently detect natural radon accumulating in a box without the expensive photon counting systems. This prototype system provides continuous measurements at high temporal rates (minute or less) and for long time periods autonomously. More studies will be carried to optimize the correct integration and dead time, to improve sensitivity using a mirror and to further characterize the response of this sensor to ambient radon.

\section{Acknowledgements}

This work is financed by the North Portugal Regional Operational Programme (NORTE 2020), under the PORTUGAL 2020 Partnership Agreement, and through the European Regional Development Fund (ERDF) within the project «Coral - Sustainable Ocean Exploitation: Tools and Sensors/NORTE-01-0145-FEDER000036».

\section{References}

[1] G. Friedlander, J. W. Kennedy, E. S. Macias, and J. M. Miller, Nuclear and Radiochemistry 3rd Ed (Wiley Interscience, 1981 ), Vol. 35 .

[2] J. H. Hendry, S. L. Simon, A. Wojcik, M. Sohrabi, E. Cardis, D. Laurier, M. Tirmarche, and I. Hayata, "Human exposure to high natural background radiation: what can it teach us about radiation risks?," J. Radiol. Prot. 29, A29-A42 (2009).

[3] S. M. Barbosa, R. V. Donner, and G. Steinitz, "Radon applications in geosciences - Progress \& perspectives," Eur. Phys. J. Spec. Top. 224, 597-603 (2015).

[4] D. Sporea, A. Sporea, S. O'Keeffe, D. McCarthy, and E. Lewis, "Optical Fibers and Optical Fiber Sensors Used in Radiation Monitoring," Sel. Top. Opt. Fiber Technol. 607-652 (2012).

[5] A. I. de Andrés, Ó. Esteban, and M. Embid, "Optical fiber sensor for low dose gamma irradiation monitoring," Sixth Eur. Work. Opt. Fibre Sensors 9916, 99160D (2016).

[6] C. H. Park, J. H. Moon, and B. K. Seo, "Development and characterization of the integrated fiber-optic sensor for remote detection of alpha radiation," J. Korean Phys. Soc. 63, 1720-1723 (2013).

[7] S. O’Keeffe, C. Fitzpatrick, E. Lewis, and A. I. Al $\square$ Shamma'a, "A review of optical fibre radiation dosimeters," Sens. Rev. 28, 136$142(2008)$

[8] S. Yamamoto, Y. Yoshida, and T. Iida, "Development of an underground radon detector using an optical fiber," IEEE Trans. Nucl. Sci. 50, 987-990 (2003).

[9] M. Mirhabibi, A. Negarestani, M. A. Bolorizadeh, M. R. Rezaei, and A. Akhound, "A new approach for radon monitoring in soil as an earthquake precursor using optical fiber," J. Radioanal. Nucl. Chem. 301, 207-211 (2014).

[10] A. Appleby and I. S. Kim, "Measuring ambient radon using a scintillating optical fiber bundle," Radiat. Phys. Chem. 55, 749-751 (1999).

[11] I. S. Kim, I. J. Lee, A. Appleby, E. A. Christman, M. J. Liepmann, and G. H. Sigel, "A new approach to monitoring radon and radon progeny using a glass scintillator in a fiber bundle structure," Nucl. Inst. Methods Phys. Res. A 356, 537-543 (1995).

[12] K. K. Mitev, "Measurement of $222 \mathrm{Rn}$ by absorption in plastic scintillators and alpha/beta pulse shape discrimination," Appl. Radiat. Isot. 110, 236-243 (2016). 